\title{
Photodissociation of water induced by a long UV pulse and probed by high-energy-resolution x-ray-absorption spectroscopy
}

\author{
Viktoriia Savchenko $\odot,{ }^{1,2}$ Ji-Cai Liu $\odot,{ }^{3,}{ }^{*}$ Michael Odelius $\odot,{ }^{4}$ Nina Ignatova $\odot,{ }^{1}$ Faris Gel'mukhanov, ${ }^{2,1,5}$ \\ Sergey Polyutov $\odot,{ }^{1}$ and Victor Kimberg $\odot^{2,1, \uparrow}$ \\ ${ }^{1}$ International Research Center of Spectroscopy and Quantum Chemistry, Siberian Federal University, 660041 Krasnoyarsk, Russia \\ ${ }^{2}$ Department of Theoretical Chemistry and Biology, KTH Royal Institute of Technology, 10691 Stockholm, Sweden \\ ${ }^{3}$ Department of Mathematics and Physics, North China Electric Power University, 102206 Beijing, China \\ ${ }^{4}$ Department of Physics, AlbaNova University Center, Stockholm University, SE-106 91 Stockholm, Sweden \\ ${ }^{5}$ Institute for Methods and Instrumentation in Synchrotron Radiation Research, Helmholtz-Zentrum Berlin für Materialien und Energie, \\ Albert-Einstein-Strasse 15, 12489 Berlin, Germany
}

(Received 12 May 2021; revised 29 July 2021; accepted 19 August 2021; published 13 September 2021)

\begin{abstract}
A UV-pump x-ray-probe (UVX-PP) experiment for probing UV initiated dissociation of the water molecule with long pulses is proposed on the basis of theoretical simulations. With long overlapping UV and x-ray pulses, we can obtain high-spectral-resolution x-ray-absorption or ionization spectra, containing detailed information about dynamics in the intermediate valence-excited state. The dynamics can be influenced with frequency detuning of the pump UV pulse, which acts as a camera shutter by regulating the duration of the UVX-PP process. Thereby, this UVX-PP setup gives access to ultrafast dynamics of the nuclear wave packet without experimentally challenging requirements of ultrashort pulses and controlled delay times. In a case study of the water molecule, we focus on the lowest UVX-PP channel $\left(1 b_{1} \rightarrow 4 a_{1}, 1 a_{1} \rightarrow 1 b_{1}\right)$ where both intermediate valence-excited and final core-excited states are dissociative. The variation of the UVX-PP duration, controlled by the UV detuning, and different dispersion laws of so-called molecular and atomic bands allow one to study the dynamics of fragmentation of the water molecule in the intermediate state. A feature is that the long lifetime of intermediate valence-excited states opens a door for studies of photoinduced dissociation of polyatomic molecules with heavy fragments.
\end{abstract}

DOI: 10.1103/PhysRevA.104.032816

\section{INTRODUCTION}

Time resolved pump-probe spectroscopy is a powerful tool for tracking ultrafast electron and nuclear dynamics. Due to the fast development of new light sources in the x-ray diapason, pump-probe spectroscopy is moving rapidly into the short-wavelength range [1-3]. For example, high flux femtosecond $\mathrm{x}$-ray pulses allow time resolved $\mathrm{x}$-ray-diffraction measurements which give insight into phase-transition dynamics in solids induced by excitation of large-amplitude coherent optical phonons [4-6]. Pump-probe spectroscopy was initially enabled by progress in the field of optical laser physics. High time resolution is often achieved with optimally shaped, ultrashort laser pulses [7], but this is accomplished at the expense of spectral resolution due the energy-time uncertainty principle. As an alternative to short laser pulses [8],

*jicailiu@ncepu.edu.cn

${ }^{\dagger}$ kimberg@kth.se

Published by the American Physical Society under the terms of the Creative Commons Attribution 4.0 International license. Further distribution of this work must maintain attribution to the author(s) and the published article's title, journal citation, and DOI. Funded by Bibsam. setups operated with long pulses [9] can give high spectral resolution, which will be utilized in this paper.

Pump pulses often involve infrared (IR), optical, or ultraviolet (UV) pulses, while depending on the source the probe pulses vary from terahertz to $\mathrm{x}$-ray wavelengths. Time delayed, Fourier-transform limited, and phase controlled IR or UV pulses in table top experiments [10] can be used to track vibrational and electronic states. IR and x-ray pulses can be generated from synchrotrons [11,12], higher harmonic generation [13], or free-electron lasers (XFELs) [14]. Also xray pump-X-ray-probe experiments are possible in which high spectral resolution can be obtained even for short stochastic XFEL pulses with limited spectral coherence using the covariance-mapping technique [15].

One of nature's most fundamental processes is chemical reactions in which chemical bonds in molecules rearrange to form new compounds. Mapping the evolution of electronic structure and nuclear dynamics is the key to explain mechanisms of chemical reactions. Quite often the photoexcitation is accompanied by the fragmentation. The study of the dynamics of light-induced fragmentation or photolysis of the molecules constitutes one of the important directions of ultrafast photochemistry $[4,16]$. Time resolved measurements can be performed directly by varying the delay time between short pump and probe pulses in conventional time resolved pumpprobe spectroscopy with limited spectral resolution due to 
short pulse duration. For example, photodissociation dynamics of OCS into $\mathrm{CO}$ and $\mathrm{S}$ fragments was observed through time delayed strong-field ionization and imaging of the kinetic energy of the resulting $\mathrm{CO}^{+}$and $\mathrm{S}^{+}$fragments [17]. Time resolved VUV photoelectron spectroscopy was used to map the entire occupied valence electronic structure of photoexcited gas-phase $\mathrm{Br}_{2}$ molecules during dissociation $[18,19]$.

The UV-pump x-ray-probe (UVX-PP) technique presents an alternative opportunity to study nuclear dynamics in valence-excited states using long pulses which preserve high spectral resolution. This was recently demonstrated for the UVX-PP process between bound states of the $\mathrm{CO}$ molecule [20]. However, the excitation of molecules in repulsive states is a rather common phenomenon. Contrary to the earlier studied pump-probe scheme with the bound states [20], the photofragmentation brings a qualitatively new element, namely, the opportunity to map the evolution of the molecule from the equilibrium through the possibly transient state to the region of fragmentation. In the UVX-PP process the pump UV pulse dissociates the molecule into molecular fragments and the probe $\mathrm{x}$-ray pulse provides a way for their identification using element and state sensitivity of $\mathrm{x}$-ray absorption. This method is a valuable tool for the study of mechanisms of fast photochemical reactions.

Similar to resonant inelastic X-ray scattering (RIXS) [4] the UVX-PP method allows us to study in detail the dynamics of the fragmentation. As we will show below, this method allows us to trace in time development of two spectral bands-one of the intact molecules ("molecular" band) and dissociation fragments ("atomic" or "fragment" band). Contrary to RIXS with the short lifetime of the intermediate core-excited state, the long lifetime of the intermediate valence-excited state allows us to study the fragmentation into the heavy fragments. Due to the long lifetime of the intermediate valence-excited state, the nuclear wave packet experiences dramatic changes in the course of the UV-induced fragmentation. Let us single out two important ingredients which couple dynamics and the spectrum. The first one is that the shape of the wave packet in different instances leads to specific spectral features like molecular and atomic bands. The second key point is the internal timer of the UVX-PP process with the timing regulated by the duration time $\tau$. This time, controlled by the detuning $\Omega_{\mathrm{UV}}$ from the UV absorption resonance, arises because of the dephasing $\exp \left(i \Omega_{\mathrm{Uv}} t\right)$ of the amplitude in the process, which quenches the long-time $t>\tau$ contributions similar to RIXS [21].

The molecular band is formed by the probe pulse generating $\mathrm{x}$-ray transitions near the equilibrium geometry, while the atomic band is mainly due to core excitations of the fragments of dissociation. We have already encountered such a structure in similar processes, namely, RIXS and resonant Auger scattering (RAS) against dissociative intermediate core-excited states $[4,22,23]$. Due to different dispersion of the atomic and molecular bands as a function of excitation energy, their spectral features can be separated. However, quite often the atomic peak in RIXS and RAS is rather weak because the coreexcited molecule does not have time to entirely dissociate during the short lifetime of the intermediate core-excited state. Hence, it is very difficult to track the dissociation into heavy fragments, which is a common relaxation pathway for many core excitations. This drawback in RIXS and RAS is absent in UVX-PP due to a much longer lifetime of the intermediate valence-excited state. Another advantage of the proposed UV $\mathrm{x}$-ray pump-probe method is that one can use ordinary synchrotron light sources with pulses longer than $1 \mathrm{ps}$. There is also a direct approach to correlate the relaxation pathways and the fragments of the dissociation using the time-of-flight and photoelectron-photoion coincidence measurements [24-26]. However, contrary to the UVX-PP technique, these methods cannot identify the electron-vibrational state of the fragment.

To exemplify the concept of UVX-PP for dissociative states, we perform spectrum simulations for the water molecule, where the UV excitation $1 b_{1} \rightarrow 4 a_{1}$ of the molecule into the lowest valence-excited state $\tilde{A}\left(1 b_{1}^{-1} 4 a_{1}^{1}\right)$ is followed by a x-ray probe transition $1 a_{1} \rightarrow 1 b_{1}$ to the coreexcited state $1 a_{1}^{-1} 4 a_{1}^{1}$. Here, each molecular orbital (MO) involved in the transitions is denoted by its irreducible representation in the $C_{2 v}$ point group of the ground-state water molecule. The oxygen $1 s$ core orbital is denoted $1 a_{1}$, whereas in the valence $1 b_{1}$ is the highest occupied lone-pair MO and $4 a_{1}$ is the lowest unoccupied antibonding MO. A comprehensive review of UV absorption transitions in the water molecule can be found in Ref. [27].

The first valence-excited state $\tilde{A}\left(1 b_{1}^{-1} 4 a_{1}^{1}\right)$ is dissociative with the same dissociation limit as the ground state $X^{1} A_{1}$ and has been extensively studied theoretically $[16,28-36]$. The first core-excited state $1 a_{1}^{-1} 4 a_{1}^{1}$ and related two-dimensional potential-energy surfaces of ground, valence-excited, and core-excited states were computed earlier in Refs. [28-31] and studied experimentally in Ref. [37]. One should mention also another-IR-pump x-ray-probe—scheme [38,39] applied to study the water molecule. This method allows us to probe nuclear dynamics in the core-excited state of vibrationally excited molecules using one or two differently polarized coherent IR pulses [40].

Our paper has the following structure. We begin in Sec. II with the outline of the employed model and the time-dependent wave-packet formulation of the pump-probe process. Section III is devoted to the computational details. Results of the simulations are collected and discussed in Sec. IV. We summarize our findings in Sec. V.

\section{DYNAMICAL THEORY OF THE UVX-PP PROCESS}

In this paper, we will study the interaction of molecules with two long overlapping UV and x-ray pulses (we use atomic units throughout the paper if it is not stated otherwise):

$$
\mathcal{E}_{\mathrm{UV}}=\frac{1}{2} E_{\mathrm{UV}} \mathbf{e}_{\mathrm{UV}} e^{i \omega_{\mathrm{UV}} t}+\text { c.c., } \quad \mathcal{E}_{X}=\frac{1}{2} E_{X} \mathbf{e}_{X} e^{i \omega_{X} t}+\text { c.c. }
$$

These fields play different roles. The pump UV pulse $\left(E_{\mathrm{UV}}\right.$, $\left.\mathbf{e}_{\mathrm{UV}}, \omega_{\mathrm{UV}}\right)$ excites the molecule from the ground state zero to the valence-excited state 1 and triggers the propagation of the nuclear wave packet in the field of the potential $U_{1}(R)$. The x-ray pulse with the amplitude $E_{X}$, polarization vector $\mathbf{e}_{X}$, and frequency $\omega_{X}$ promotes the molecule into a core-excited state 2 , thereby generating an x-ray-absorption spectrum of valence-excited molecules which probes the dynamics of the nuclear motion in the intermediate state 1 . In the specific 


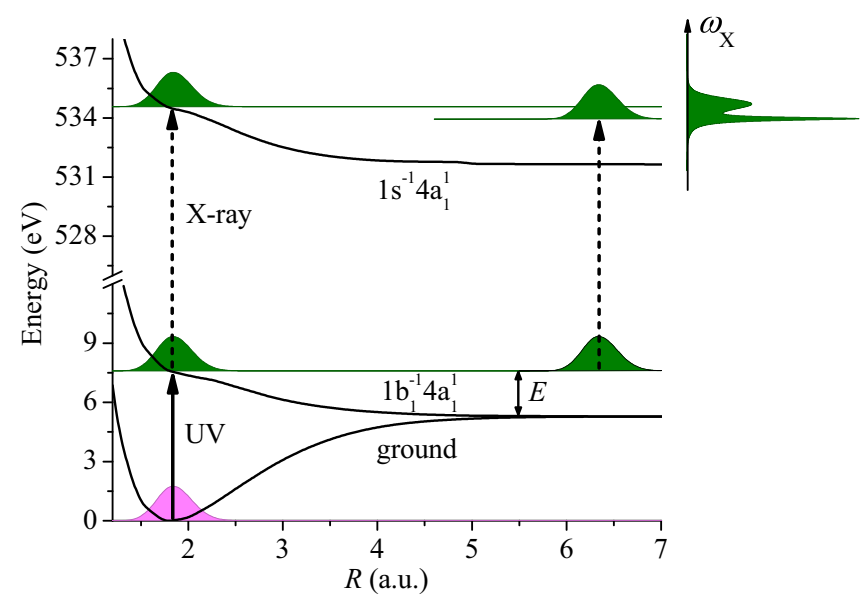

FIG. 1. UV+x-ray pump-probe scheme exemplified for the lowest UVX-PP channel of the $\mathrm{H}_{2} \mathrm{O}$ molecule: UV transition $1 b_{1} \rightarrow 4 a_{1}$ [27] followed by x-ray transition $1 a_{1} \rightarrow 1 b_{1}$. The details of calculations of potential-energy surfaces depicted in this figure are outlined in Sec. III.

process for the water molecule (see Fig. 1)

$$
\begin{aligned}
\omega_{\mathrm{UV}}+\left|X^{1} A_{1}\right\rangle & \rightarrow\left|\tilde{A}\left(1 b_{1}^{-1} 4 a_{1}^{1}\right)\right\rangle \rightarrow \omega_{X}+\left|\tilde{A}\left(1 b_{1}^{-1} 4 a_{1}^{1}\right)\right\rangle \\
& \rightarrow\left|1 a_{1}^{-1} 4 a_{1}^{1}\right\rangle
\end{aligned}
$$

The theory of this pump-probe process was developed earlier and can be found in Ref. [20]. In short, the cross section of the UVX-PP process

$$
\sigma=\varrho \sigma_{\text {nucl }}, \quad \varrho \propto \overline{\left|\left(\mathbf{e}_{\mathrm{UV}} \cdot \mathbf{d}_{01}\right)\left(\mathbf{e}_{X} \cdot \mathbf{d}_{12}\right)\right|^{2}}
$$

depends on the polarization of applied fields through the prefactor $\varrho$, where the overline implies an average over molecular orientations [4,22]. The dipole moments on the UV $\left[\mathbf{d}_{01}=\right.$ $\left.\left\langle 1 b_{1}|\mathbf{r}| 4 a_{1}\right\rangle=\left(\mathbf{d}_{01}^{(x)}, 0,0\right)\right]$ and $\mathrm{x}$-ray $\left[\mathbf{d}_{12}=\left\langle 1 a_{1}|\mathbf{r}| 1 b_{1}\right\rangle=\right.$ $\left.\left(\mathbf{d}_{12}^{(x)}, 0,0\right)\right]$ transitions are perpendicular to the molecular $(z, y)$ plane because of the orthogonal orientation of the lone pair $1 b_{1}$ orbital.

The UVX-PP spectrum is described by the factor $\sigma_{\text {nucl }}$ [20]:

$$
\begin{aligned}
\sigma_{\text {nucl }} & =\frac{1}{\pi} \operatorname{Re} \int_{0}^{\infty} d t \sigma(t) e^{i\left(\omega_{\mathrm{Uv}}+\omega_{X}-\omega_{20}+\epsilon_{0}+i \Gamma\right) t}, \\
\sigma(t) & =\langle\Psi(0) \mid \Psi(t)\rangle, \quad|\Psi(t)\rangle=e^{-i h_{2} t} d_{21}|\Psi(0)\rangle, \\
|\Psi(0)\rangle & =\int_{0}^{\infty} d t e^{-\frac{t}{\tau_{C}}}\left|\psi_{1}(t)\right\rangle, \quad\left|\psi_{1}(t)\right\rangle=e^{-i h_{1} t} d_{10}|0\rangle .
\end{aligned}
$$

Here $h_{n}=K+U_{n}(R)-U_{n}^{\min }$ is the nuclear Hamiltonian of the $n$th electronic state. We omit here the derivation of Eq. (4) because it is very similar to the case of RIXS [41]. The spectrum [see Eq. (4)] depends on the dynamics of the nuclear wave packets in the intermediate valence-excited $\left|\psi_{1}(t)\right\rangle$ and final core-excited $|\Psi(t)\rangle$ states. This dynamics is controlled by the complex time

$$
\tau_{C}=\frac{1}{\gamma-i\left(\omega_{\mathrm{UV}}-\omega_{10}+\epsilon_{0}\right)} .
$$

It is natural to name the absolute value $\tau=\left|\tau_{C}\right|$ of $\tau_{C}$ as the duration of the UVX-PP process, which can be controlled by detuning from the UV absorption resonance, $\Omega_{\mathrm{UV}}=\omega_{\mathrm{UV}}-$ $\omega_{10}+\epsilon_{0}$. The duration time (see also Ref. [20])

$$
\tau=\left|\tau_{C}\right|=\frac{1}{\sqrt{\Omega_{\mathrm{UV}}^{2}+\gamma^{2}}}
$$

effectively plays the same role as the pump-probe delay time in conventional time resolved spectroscopy. Here we define $\omega_{m n}=U_{m}^{\min }-U_{n}^{\min }$ as the difference between the bottom of the potential wells $U_{m}(R)$ and $U_{n}(R)$. The dynamics of the studied process is characterized by two relaxation time constants, namely, the lifetime broadenings (half width at half maximum) of the valence $\gamma$ and core-excited $\Gamma$ states. The prefactor $\varrho$ (3) is not included in simulations, because the spectral shape of the UVX-PP cross section does not depend on the polarization vectors. In the case of water, the dissociative intermediate and final states make it numerically convenient to use a time-dependent representation $[20,28,41,42]$ for the nuclear part (4) of the UVX-PP cross section. The time-dependent representation for the cross section (4) together with the duration time (6) has also the advantage that it shows how the spectrum is affected by the dynamics of nuclear wave packet $\left|\psi_{1}(t)\right\rangle$ as controlled by the frequency detuning $\Omega_{\mathrm{UV}}$ via the factor $\exp \left(-t / \tau_{C}\right)$. This important aspect will be elucidated in detail in Sec. IV.

\section{DETAILS OF SIMULATIONS}

The potential-energy surfaces of $\mathrm{H}_{2} \mathrm{O}$ (Fig. 1) are derived from calculations [31] carried out in the restricted active space self-consistent field [43] framework in the MOLCAS package [44]. Other details can be found in Ref. [31]. Since the UV transition occurs near the equilibrium distance $R_{0}$, where the transition dipole moment $\mathbf{d}_{01}$ is almost constant, we neglect its $R$ dependence. Also, the $R$ dependence of the transition dipole moment of $\mathrm{x}$-ray excitation $\mathbf{d}_{12}$ is negligible.

During the long lifetime $(1 / \gamma)$ of the valence-excited state $1 b_{1}^{-1} 4 a_{1}^{1}$, the wave packet propagates a large distance, $R>$ $R_{0}+v / \gamma \approx 26$ a.u. Here $v \approx \sqrt{2 E / m_{H}}$ is the velocity of the leaving hydrogen atom, where the kinetic energy $E$ is defined in Fig. 1. In order to get full convergence of the results of the simulations, we propagate the wave packet $\left|\psi_{1}(t)\right\rangle$ up to $R=95$ a.u. The experimental value $[28,45]$ of the lifetime broadening of the core-excited state, $\Gamma=0.079 \mathrm{eV}$ $(1 / \Gamma=8.33 \mathrm{fs})$, was used in the calculations. For the lifetime broadening of the valence-excited state we used only a reasonable estimation, $\gamma=0.01 \mathrm{eV}(1 / \gamma=65.8 \mathrm{fs})$. In a real system the lifetime of a lower valence-excited state is usually much longer, which results in smaller lifetime broadening, thus improving the spectral resolution of the UVX-PP technique. Here we consider the case of weak long UV and x-ray pulses, which implies that the pulses cannot make any sufficient change in the populations and that their duration is sufficiently longer as compared to all relaxation times of the system. Under these conditions, the actual pulse parameters do not play any role for the simulation. In order to fulfill the above stated criteria for the pulse parameters in experiment the pulse durations must be chosen so that $\tau_{X}, \tau_{\mathrm{UV}} \gg 1 / \Gamma, 1 / \gamma \gg 100 \mathrm{fs}$; for the pulse intensities the corresponding Rabi frequencies must satisfy the following 

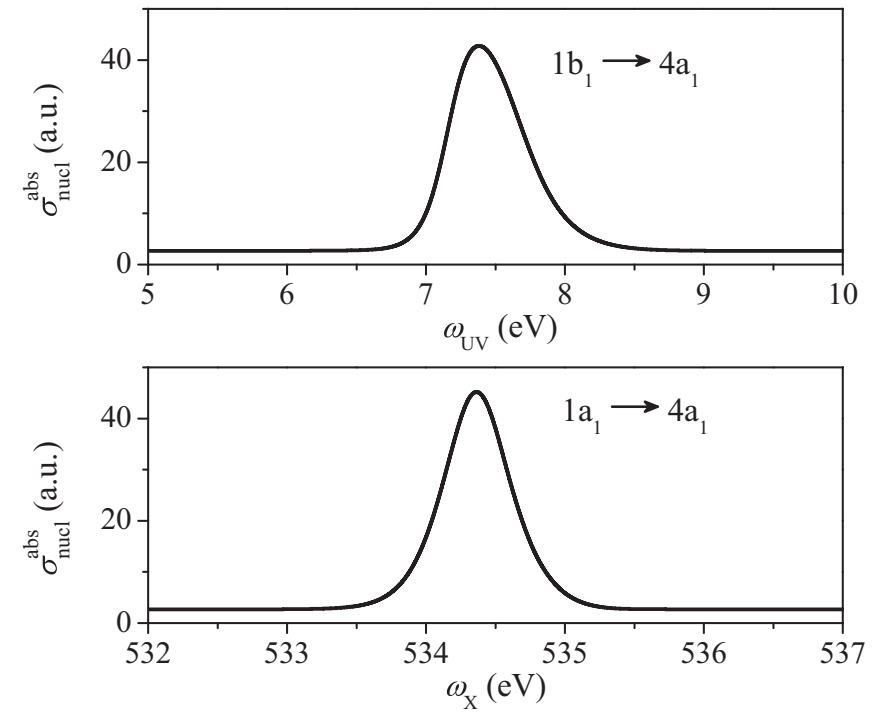

FIG. 2. Theoretical UV and x-ray-absorption profiles of $\mathrm{H}_{2} \mathrm{O}$. The experiments give the following value of the peak positions: $7.447 \mathrm{eV}$ for $1 b_{1} \rightarrow 4 a_{1} \mathrm{UV}$ absorption [27] and $534 \mathrm{eV}$ for $1 a_{1} \rightarrow$ $4 a_{1}$ x-ray absorption [37].

condition: $\left|E_{\mathrm{Uv}} d_{01}\right|,\left|E_{X} d_{12}\right| \ll \Gamma, \gamma$. Given that the transition dipole moment of the core-to-valence transition is 0.08 a.u. [31] the laser intensity must be limited by $\ll 10^{12} \mathrm{~W} / \mathrm{cm}^{2}$. Let us note that deviation from the Gaussian pulse envelope may result in the formation of weak side spectral structures as it was shown for the case of a rectangular pulse for $\mathrm{x}$-ray absorption [46].

The calculation of the UVX-PP cross section $\sigma_{\text {nucl }}$ (4) was performed using wave-packet software [47] employing a direct solution of the time-dependent Schrödinger equation in Cartesian coordinates with a split-time operator algorithm [48]. Before concluding this section, we should notice that the dynamics of the UVX-PP process is sensitive $[28,41,42]$ to the spectral shape of the UV absorption $0 \rightarrow 1$,

$$
\begin{aligned}
\sigma_{\text {nucl }}^{\mathrm{abs}} & =\frac{1}{\pi} \operatorname{Re} \int_{0}^{\infty} d t \sigma_{1}(t) e^{i\left(\omega_{\mathrm{Uv}}-\omega_{10}+\epsilon_{0}+i \gamma\right) t}, \\
\sigma_{1}(t) & =\left\langle 0 \mid \psi_{1}(t)\right\rangle,
\end{aligned}
$$

as well as to the $\mathrm{x}$-ray absorption $0 \rightarrow 2$, which is defined by the same equation after replacement of the UV frequency by a x-ray frequency $\left(\omega_{\mathrm{UV}} \rightarrow \omega_{X}\right)$, the valence excitation by the core excitation $(1 \rightarrow 2)$, and a corresponding replacement of the lifetime broadening $(\gamma \rightarrow \Gamma)$.

\section{RESULTS AND DISCUSSION}

\section{A. Photodissociation dynamics of water}

Because both the intermediate valence-excited and the final core-excited states are dissociative the UV and x-rayabsorption profiles computed using Eq. (7) show broad peaks (Fig. 2). At the UV resonance ( $\left.\Omega_{\mathrm{UV}}=0\right)$, the UVX-PP spectrum [see Eq. (4)] exhibits a narrow atomic peak (Fig. 3), which is much stronger than the broad molecular band. This differs qualitatively from the RIXS spectra of water [31] and methanol [41] where the intensity of the molecular band is

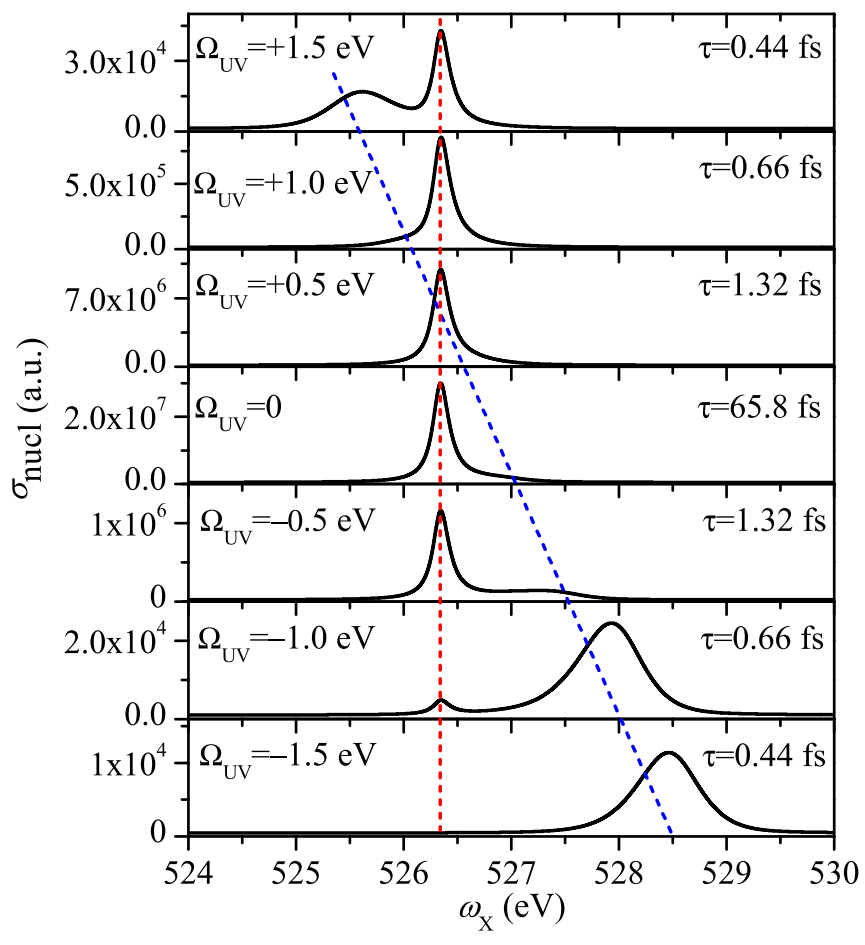

FIG. 3. Theoretical UVX-PP spectra (4) of $\mathrm{H}_{2} \mathrm{O}$ for different detuning $\Omega_{\mathrm{UV}}=\omega_{\mathrm{UV}}-\omega_{10}+\epsilon_{0}$. One can see the anti-Raman dispersion (8) for the molecular band and nondispersive for the atomic peak, which vanishes for large $\left|\Omega_{\mathrm{UV}}\right|$. Guidelines for anti-Raman dispersion (blue) and nondispersive (red) are included. The UVX-PP spectra show the gradual quenching of the nondispersive (8) atomic peak (red) with an increase of $\left|\Omega_{\mathrm{UV}}\right|$ due to shortening of the duration $\tau$ (6). When the UVX-PP process becomes fast we have an almost instantaneous transition from ground to final state, $0 \rightarrow 2$. Due to this, the dispersive molecular (blue) band (8) converges to the broadband which mimics the shape of x-ray-absorption spectroscopy $1 a_{1} \rightarrow 4 a_{1}$ shown in Fig. 2 .

comparable with that of the atomic peak. The reason for this is the short lifetime of the intermediate core-excited state in RIXS in comparison with the long lifetime of the intermediate valence-excited state in the UVX-PP process which makes the probability of fragmentation much larger in UVX-PP than in RIXS, namely, $\Gamma / \gamma$ times larger. As it was pointed out earlier [20], the dynamics of the UVX-PP process is characterized not only by the lifetime of intermediate state $1 / \gamma$ but also by the detuning from the top of UV absorption $\Omega_{\mathrm{UV}}=\omega_{\mathrm{UV}}-$ $\omega_{10}+\epsilon_{0}$. Above, Eqs. (4) and (5) contain the characteristic time constant $\tau=\left|\tau_{C}\right|$ [see Eq. (6)] which characterizes the duration of the studied process (similar to RIXS [21,22]). Figure 3 shows that the dependence of the UVX-PP spectrum on the detuning is not the same for positive and negative $\Omega_{\mathrm{UV}}$. The reason for this is the asymmetry of the spectrum with respect to the point of the vertical transition in potential-energy curve $U_{1}(R)$ (see state $1 b_{1}^{-1} 4 a_{1}^{1}$ in Fig. 1).

The shortening of the UVX-PP duration with increase of $\left|\Omega_{U V}\right|$ explains the quenching of the narrow atomic peak when the frequency of the pump UV radiation is tuned away from the resonance (see Fig. 3). The observed quenching of the atomic peak is, however, only one part of the dynamical 
effects found in our simulations. One can see also that the molecular and atomic bands

$$
\omega_{X}^{(\mathrm{mol})} \approx-\omega_{\mathrm{UV}}+\omega_{20}\left(R_{0}\right), \quad \omega_{X}^{(\mathrm{at})} \approx \omega_{21}(\infty)=\mathrm{const}
$$

depend very differently on the frequency of the UV field (Fig. 3), showing anti-Raman dispersion and nondispersive behavior, respectively. Here $\omega_{m n}(R)=U_{m}(R)-U_{n}(R)$. This behavior can be understood by examining the energy conservation laws for the molecular band, formed near equilibrium $\left[\omega_{\mathrm{UV}}+U_{0}\left(R_{0}\right)+\omega_{X} \approx U_{2}\left(R_{0}\right)\right]$, and for the atomic peak $\left[\omega_{X}+U_{1}(\infty) \approx U_{2}(\infty)\right]$, formed in the asymptotic region where the potentials $U_{1}(R)$ and $U_{2}(R)$ are both flat (see Fig. 1). One should notice that the anti-Raman dispersion law is strictly fulfilled only when the UV frequency $\omega_{\mathrm{UV}}$ is tuned beyond the $0 \rightarrow 1 \mathrm{UV}$ resonance (similar to RIXS [22]).

\section{B. Photodissociation dynamics of polyatomic molecules with heavy fragments}

The suggested UVX-PP technique is nicely suited for studying dynamics of the photoinduced fragmentation of polyatomic molecules with heavy fragments of the dissociation. Indeed, the dynamics of the dissociation channels leading to formation of heavy fragments cannot be detected by RIXS, because the lifetime of the intermediate core-excited state ( $\lesssim 1 \mathrm{fs})$ is much shorter than the fragmentation time [4]. This drawback is absent in the UVX-PP technique due to the much longer lifetime of the intermediate valence-excited state ( $\gtrsim 100 \mathrm{fs}$ ).

Let us point out another advantage of the suggested UVXPP method for fragmentation dynamics studies. In the case of photodissociation of a polyatomic molecule by visible or UV light [24,25], the tunability of synchrotron or XFEL radiation allows us to separate probe $\mathrm{x}$-ray spectra from different dissociation fragments due to element selectivity of x-ray spectroscopy. The first showcase is an important reaction of the formation of tropospheric ozone [49]:

$$
\mathrm{NO}_{2}+\omega_{\mathrm{UV}} \rightarrow \mathrm{NO}+\mathrm{O} .
$$

When X-ray radiation is tuned near the $\mathrm{N} \mathrm{K}$ edge one can probe in time the dissociation dynamics of the NO fragment. In the case when $x$ rays are tuned near the $\mathrm{O} K$ edge the probe $\mathrm{x}$-ray-absorption spectrum includes both $\mathrm{NO}$ and the oxygen atom x-ray-absorption spectra contributions. However, these probe signals can be distinguished since the first $\mathrm{x}$-ray-absorption spectroscopy resonances of the NO molecule $(531.1 \mathrm{eV})[50]$ and of the $\mathrm{O}$ atom $(527 \mathrm{eV})$ [51] are separated by approximately $4 \mathrm{eV}$.

Another example is UV-induced dissociation dynamics of nitromethane $\mathrm{CH}_{3} \mathrm{NO}_{2}$ [52]. Excitation to the lowest band $\omega_{\mathrm{UV}} \approx 6.3 \mathrm{eV}$ corresponds to the $n \rightarrow \pi^{*}$ transition localized on the $\mathrm{NO}_{2}$ moiety, thus the main photodissociation channel is [52]

$$
\mathrm{CH}_{3} \mathrm{NO}_{2} \rightarrow \mathrm{CH}_{3}+\mathrm{NO}_{2} .
$$

The dynamics of the reaction can be probed by the UVX-PP technique using $\mathrm{x}$ rays tuned in the vicinity of either the $\mathrm{C}$ $\mathrm{K}$ edge of $\mathrm{CH}_{3}$ or the $\mathrm{N} \mathrm{K}(\mathrm{O} \mathrm{K})$ edge of $\mathrm{NO}_{2}$ fragments similar to Fig. 3. The element selectivity of $\mathrm{x}$ rays gives a simple and powerful tool to identify fragments of dissociation as well as the electronic states of the fragments because $\mathrm{x}$ ray-absorption spectra of $\mathrm{CH}_{3}$ and $\mathrm{NO}_{2}$ are sensitive to their electron-vibrational excitation $[4,53]$.

\section{SUMMARY}

We have explored the theoretical foundations for using long overlapping UV and x-ray pulses for obtaining a highresolution $\mathrm{UV}+\mathrm{x}$-ray pump-probe spectrum of gas-phase water. We have chosen a channel in which first the UV pulse excites the electron from highest occupied $1 b_{1} \mathrm{MO}$ to lowest unoccupied $4 a_{1} \mathrm{MO}$, then the x-ray pulse promotes the $O 1 s$ electron to the created hole in the lone pair $1 b_{1}$ orbital. The choice of these channels is twofold. First of all, this allows us to avoid spectral overlap of the UVX-PP signal with the conventional X-ray absorption from the ground state. Secondly, our paper sheds light on the advantages of the suggested pump-probe scheme in studies of molecular fragmentation because both intermediate $\left(1 b_{1}^{-1} 4 a_{1}\right)$ and final $\left(1 a_{1}^{-1} 4 a_{1}\right)$ states are dissociative. One of the main advantages of the proposed scheme for UVX-PP spectroscopy is that a $\mathrm{O}-\mathrm{H}$ bond in the water molecule is almost completely broken in the long-lived, dissociative valence-excited state $1 b_{1}^{-1} 4 a_{1}$. This drastically enhances (by $\Gamma / \gamma$ times) the intensity of the $\mathrm{X}$-ray-probe signal from the fragment of the dissociation in comparison with akin processes, like RIXS [31] and RAS [4] with the short lifetime of the intermediate state. This circumstance together with the very different dispersion of the so-called molecular and atomic bands and variable duration of the process controlled by the frequency of the UV field opens the door to study photoinduced dissociation on a longer timescale as in the case of heavy dissociation fragments. The detection of heavy products of the dissociation is impossible in RIXS and RAS spectroscopy due to the short lifetime of core-excited states.

\section{ACKNOWLEDGMENTS}

The reported study was funded by the Russian Science Foundation, Project No. 21-12-00193. J.-C.L. acknowledges support by the National Natural Science Foundation of China under Grants No. 11974108 and No. 11574082, and the Natural Science Foundation of Shandong Province under Grant No. ZR2019MA020. M.O. acknowledges funding from the European Union's Horizon 2020 research and innovation program under Marie Skłodowska-Curie Grant No. 860553 and the Carl Tryggers Foundation (Contract No. CTS18:285). F.G. acknowledges support from the Helmholtz Virtual Institute VI419 "Dynamic Pathways in Multidimensional Landscapes". N.I. acknowledges funding from the Foundation for the Advancement of Theoretical Physics and Mathematics "BASIS" (Grant No. 191-4-66-1). Support from the Swedish Research Council, Project No. 2019-03470, is acknowledged. The calculations were partially enabled by resources provided by the Swedish National Infrastructure for Computing, partially funded by the Swedish Research Council through Grant No. 2018-05973. 
[1] M. Kowalewski, K. Bennett, and S. Mukamel, Monitoring nonadiabatic avoided crossing dynamics in molecules by ultrafast x-ray diffraction, Struct. Dyn. 4, 054101 (2017).

[2] R. Mincigrucci, M. Kowalewski, J. R. Rouxel, F. Bencivenga, S. Mukamel, and C. Masciovecchio, Impulsive UV-pump/x-ray probe study of vibrational dynamics in glycine, Sci. Rep. 8, 15466 (2018).

[3] L. Young, K. Ueda, M. Gühr, P. H. Bucksbaum, M. Simon, S. Mukamel, N. Rohringer, K. C. Prince, C. Masciovecchio, M. Meyer, A. Rudenko, D. Rolles, C. Bostedt, M. Fuchs, D. A. Reis, R. Santra, H. Kapteyn, M. Murnane, H. Ibrahim, F. Légaré, M. Vrakking, M. Isinger, D. Kroon, M. Gisselbrecht, A. L'Huillier, H. J. Wörner, and S. R. Leone, Roadmap of ultrafast X-ray atomic and molecular physics, J. Phys. B 51, 032003 (2018) .

[4] F. Gel'mukhanov, M. Odelius, S. P. Polyutov, A. Föhlisch, and V. Kimberg, Dynamics of resonant X-ray and Auger scattering, Rev. Mod. Phys. 93, 035001 (2021).

[5] K. Sokolowski-Tinten, C. Blome, J. Blums, A. Cavalleri, C. Dietrich, A. Tarasevitch, I. Uschmann, E. Förster, M. Kammler, M. Horn-von-Hoegen, and D. von der Linde, Femtosecond X-ray measurement of coherent lattice vibrations near the Lindemann stability limit, Nature (London) 422, 287 (2003).

[6] S. L. Johnson, P. Beaud, C. J. Milne, F. S. Krasniqi, E. S. Zijlstra, M. E. Garcia, M. Kaiser, D. Grolimund, R. Abela, and G. Ingold, Nanoscale Depth-Resolved Coherent Femtosecond Motion in Laser-Excited Bismuth, Phys. Rev. Lett. 100, 155501 (2008).

[7] A. González-Castrillo, A. Palacios, H. Bachau, and F. Martín, Clocking Ultrafast Wave Packet Dynamics in Molecules Through UV-Induced Symmetry Breaking, Phys. Rev. Lett. 108, 063009 (2012).

[8] K. Seto, H. Yamada, T. Kobayashi, and E. Tokunaga, Demonstration of wavelength-scan-free action spectroscopy in pump/probe measurement with supercontinuum pump light, Opt. Express 27, 6976 (2019).

[9] I. Iparraguirre, I. Aramburu, J. Azkargorta, M. A. Illarramendi, J. Fernández, and R. Balda, Wavelength tuning of Titanium Sapphire Laser by its own crystal birefringence, Opt. Express 13, 1254 (2005).

[10] S. Woutersen and H. Bakker, Resonant intermolecular transfer of vibrational energy in liquid water, Nature (London) 402, 507 (1999).

[11] L. Carroll, P. Friedli, P. Lerch, J. Schneider, D. Treyer, S. Hunziker, S. Stutz, and H. Sigg, Ultra-broadband infrared pump-probe spectroscopy using synchrotron radiation and a tuneable pump, Rev. Sci. Instrum. 82, 063101 (2011).

[12] M. Burian, B. Marmiroli, A. Radeticchio, C. Morello, D. Naumenko, G. Biasiolc, and H. Amenitsch, Picosecond pump-probe x-ray scattering at the Elettra SAXS beamline, J. Synchrotron Radiat. 27, 51 (2020).

[13] A. S. Johnson, T. Avni, E. W. Larsen, D. R. Austin, and J. P. Marangos, Attosecond soft x-ray high harmonic generation, Phil. Trans. R. Soc. A 377, 20170468 (2019).

[14] E. Savelyev, R. Boll, C. Bomme, N. Schirmel, H. Redlin, B. Erk, S. Düsterer, E. Müller, H. Höppner, S. Toleikis, J. Müller, M. K. Czwalinna, R. Treusch, T. Kierspel, T. Mullins, S. Trippel, J. Wiese, J. Küpper, F. Brauße, F. Krecinic, A. Rouzée, P. Rudawski, P. Johnsson, K. Amini, A. Lauer, M.
Burt, M. Brouard, L. Christensen, J. Thøgersen, H. Stapelfeldt, N. Berrah, M. Müller, A. Ulmer, S. Techert, A. Rudenko and D. Rolles, Jitter-correction for IR/UV-XUV pump-probe experiments at the FLASH free-electron laser, New J. Phys. 19, 043009 (2017).

[15] V. Kimberg and N. Rohringer, Stochastic stimulated electronic X-ray Raman spectroscopy, Struct. Dyn. 3, 034101 (2016).

[16] R. Schinke, Photodissociation Dynamics: Spectroscopy and Fragmentation of Small Polyatomic Molecules (Cambridge University, Cambridge, England, 2009).

[17] E. T. Karamatskos, S. Yarlagadda, S. Patchkovskii, M. J. J. Vrakking, R. Welsch, J. Küpper, and A. Rouzée, Timeresolving the UV-initiated photodissociation dynamics of OCS, Faraday Discuss. 228, 413 (2021).

[18] L. Nugent-Glandorf, M. Scheer, D. A. Samuels, A. M. Mulhisen, E. R. Grant, X. Yang, V. M. Bierbaum, and S. R. Leone, Ultrafast Time-Resolved Soft X-Ray Photoelectron Spectroscopy of Dissociating $\mathrm{Br}_{2}$, Phys. Rev. Lett. 87, 193002 (2001).

[19] Ph. Wernet, M. Odelius, K. Godehusen, J. Gaudin, O. Schwarzkopf, and W. Eberhardt, Real-Time Evolution of the Valence Electronic Structure in a Dissociating Molecule, Phys. Rev. Lett. 103, 013001 (2009).

[20] J.-C. Liu, V. Savchenko, V. Kimberg, F. Gel'mukhanov, and M. Odelius, High-resolution x-ray spectra of carbon monoxide reveal ultrafast dynamics induced by long UV pulse, New J. Phys. 23, 063030 (2021).

[21] F. Gel'mukhanov, P. Sałek, T. Privalov, and Hans Ågren, Duration of x-ray Raman scattering, Phys. Rev. A 59, 380 (1999).

[22] F. Gel'mukhanov and H. Ågren, Resonant x-ray Raman scattering, Phys. Rep. 312, 87 (1999).

[23] R. Feifel, A. Baev, F. Gel'mukhanov, H. Ågren, M. N. Piancastelli, M. Andersson, G. Öhrwall, C. Miron, M. Meyer, S. L. Sorensen, A. Naves de Brito, O. Björneholm, L. Karlsson, and S. Svensson, Generalization of the duration-time concept for interpreting high-resolution resonant photoemission spectra, Phys. Rev. A 69, 022707 (2004).

[24] R. Forbes, A. E. Boguslavskiy, I. Wilkinson, J. G. Underwood, and A. Stolow, Excited state wavepacket dynamics in $\mathrm{NO}_{2}$ probed by strong-field ionization, J. Chem. Phys. 147, 054305 (2017).

[25] I. Wilkinson and B. J. Whitaker, Some remarks on the photodynamics of $\mathrm{NO}_{2}$, Annu. Rep. Prog. Chem., Sect. C 106, 274 (2010).

[26] M. N. Piancastelli, T. Marchenko, R. Guillemin, L. Journel, O. Travnikova, I. Ismail, and M. Simon, Hard X-ray spectroscopy and dynamics of isolated atoms and molecules: A review, Rep. Prog. Phys. 83, 016401 (2020).

[27] B. Wozniak and J. Dera, Light Absorption in Sea Water, Atmospheric and Oceanographic Sciences Library Vol. 33 (Springer, New York, 2007).

[28] R. C. Couto, V. V. Cruz, E. Ertan, S. Eckert, M. Fondell, M. Dantz, B. Kennedy, T. Schmitt, A. Pietzsch, F. F. Guimarães, H. Ågren, F. Gel'mukhanov, M. Odelius, V. Kimberg, and A. Föhlisch, Selective gating to vibrational modes through resonant X-ray scattering, Nat. Commun. 8, 14165 (2017).

[29] V. V. da Cruz, E. Ertan, R. C. Couto, S. Eckert, M. Fondell, M. Dantz, B. Kennedy, T. Schmitt, A. Pietzsch, F. F. Guimarães, H. Ågren, F. Gel'mukhanov, M. Odelius, A. Föhlisch, and V. Kimberg, A study of the water molecule using frequency con- 
trol over nuclear dynamics in resonant x-ray scattering, Phys. Chem. Chem. Phys. 19, 19573 (2017).

[30] S. Eckert, V. Vaz da Cruz, F. Gel'mukhanov, E. Ertan, N. Ignatova, R. C. Couto, S. Polyutov, M. Fondell, M. Dantz, B. Kennedy, T. Schmitt, A. Pietzsch, M. Odelius, and A. Föhlisch, One-dimensional cuts through multidimensional potential-energy surfaces by tunable x-rays, Phys. Rev. A 97, 053410 (2018).

[31] E. Ertan, V. Savchenko, N. Ignatova, V. Vaz da Cruz, R. C. Couto, S. Eckert, M. Fondell, M. Dantz, B. Kennedy, T. Schmitt, A. Pietzsch, A. Föhlisch, F. Gel'mukhanov, M. Odelius, and V. Kimberg, Ultrafast dissociation features in RIXS spectra of the water molecule, Phys. Chem. Chem. Phys. 20, 14384 (2018).

[32] V. Engel, R. Schinke, and V. Staemmler, An ab initio calculation of the absorption cross section of water in the first absorption continuum, Chem. Phys. Lett. 130, 413 (1986).

[33] V. Engel and R. Schinke, Isotope effects in the fragmentation of water: The photodissociation of HOD in the first absorption band, J. Chem. Phys. 88, 6831 (1988).

[34] V. Engel, R. Schinke, and V. Staemmler, Photodissociation dynamics of $\mathrm{H}_{2} \mathrm{O}$ and $\mathrm{D}_{2} \mathrm{O}$ in the first absorption band: A complete ab initio treatment, J. Chem. Phys. 88, 129 (1988).

[35] V. Engel, V. Staemmler, R. L. Vander Wal, F. F. Crim, R. J. Sension, B. Hudson, P. Andresen, S. Hennig, K. Weide, and R. Schinke, Photodissociation of water in the first absorption band: a prototype for dissociation on a repulsive potential energy surface, J. Phys. Chem. 96, 3201 (1992).

[36] D. M. Chipman, Stretching of hydrogen-bonded $\mathrm{OH}$ in the lowest singlet excited electronic state of water dimer, J. Chem. Phys. 124, 044305 (2006).

[37] L. Weinhardt, A. Benkert, F. Meyer, M. Blum, R. G. Wilks, W. Yang, M. Bär, F. Reinert, and C. Heske, Nuclear dynamics and spectator effects in resonant inelastic soft x-ray scattering of gas-phase water molecules, J. Chem. Phys. 136, 144311 (2012).

[38] N. Ignatova, V. V. Cruz, R. C. Couto, E. Ertan, A. Zimin, F. F. Guimarães, S. Polyutov, H. Ågren, V. Kimberg, M. Odelius, and F. Gel'mukhanov, Gradual collapse of nuclear wave functions regulated by frequency tuned x-ray scattering, Sci. Rep. 7, 43891 (2017).

[39] N. Ignatova, V. V. da Cruz, R. C. Couto, E. Ertan, M. Odelius, H. Ågren, F. F. Guimarães, A. Zimin, S. Polytov, F. Gel'mukhanov, and V. Kimberg, Infrared-pump-X-ray-probe spectroscopy of vibrationally excited molecules, Phys. Rev. A 95, 042502 (2017).

[40] J.-C. Liu, V. Savchenko, V. Kimberg, M. Odelius, and F. Gel'mukhanov, Polarization-sensitive IR-pump-x-ray-probe spectroscopy, Phys. Rev. A 103, 022829 (2021).
[41] V. V. da Cruz, N. Ignatova, R. C. Couto, D. Fedotov, D. R. Rehn, V. Savchenko, P. Norman, S. Polyutov, J. Niskanen, S. Eckert, R. M. Jay, M. Fondel, T. Schmidt, A. Pietzsch, A. Föhich, F. Gel'mukhanov, M. Odelius, and V. Kimberg, Nuclear dynamics in resonant inelastic x-ray scattering and x-ray absorption of methanol, J. Chem. Phys. 150, 234301 (2019).

[42] P. Sałek, F. Gel'mukhanov, and H. Ågren, Wave-packet dynamics of resonant $\mathrm{x}$-ray Raman scattering: Excitation near the $\mathrm{Cl}$ $L_{I I, I I I}$ edge of HCl, Phys. Rev. A 59, 1147 (1999).

[43] P.- $\AA$. Malmqvist, A. Rendell, and B. O. Roos, The restricted active space self-consistent-field method, implemented with a split graph unitary group approach, J. Phys. Chem. 94, 5477 (1990).

[44] F. Aquilante, J. Autschbach, R. K. Carlson, L. F. Chibotaru, M. G. Delcey, L. De Vico, I. Fdez. Galván, N. Ferré, L. M. Frutos, L. Gagliardi, M. Garavelli, A. Giussani, C. E. Hoyer, G. Li Manni, H. Lischka, D. Ma, P.-Å. Malmqvist, T. Müller, A. Nenov, M. Olivucci, T. B. Pedersen, D. Peng, F. Plasser et al., Molcas 8: New capabilities for multiconfigurational quantum chemical calculations across the periodic table, J. Comp. Chem. 37, 506 (2016).

[45] B. Brena, D. Nordlund, M. Odelius, H. Ogasawara, A. Nilsson, and L. G. M. Pettersson, Ultrafast Molecular Dissociation of Water in Ice, Phys. Rev. Lett. 93, 148302 (2004).

[46] V. Savchenko, F. Gel'mukhanov, T. Laarmann, S. Polyutov, and V. Kimberg, Dynamical phase shift in X-ray absorption and ionization spectra by two delayed x-ray laser fields, Phys. Rev. A 104, 013114 (2021).

[47] J.-C. Liu, M. C. Kohler, C. H. Keitel, and K. Z. Hatsagortsyan, Coherent $\mathrm{X}$-ray generation from below-threshold harmonics, Phys. Rev. A 84, 063817 (2011).

[48] M. D. Feit, J. A. Fleck, and A. Steiger, Solution of the Schrödinger equation by a spectral method, J. Comput. Phys. 47, 412 (1982).

[49] P. Warneck, Chemistry of the Natural Atmosphere (Academic, New York, 1999).

[50] R. Fink, A theoretical simulation of the $1 s \rightarrow 2 \pi$ excitation and deexcitation spectra of the NO molecule, J. Chem. Phys. 106, 4038 (1997).

[51] F. Frati, M. O. J. Y. Hunault, and F. M. F. de Groot, Oxygen Kedge X-ray Absorption Spectra, Chem. Rev. 120, 4056 (2020).

[52] M. S. Park, K.-H. Jung, H. P. Upadhyaya, and H.-R. Volpp, The dynamics of oxygen atom formation in the UV photodissociation of nitromethane, Chem. Phys. 270, 133 (2001).

[53] F. F. Guimarães, V. Kimberg, V. C. Felicíssimo, F. Gel'mukhanov, A. Cesar, and H. Ågren, Infrared-x-ray pump-probe spectroscopy of the NO molecule, Phys. Rev. A 72, 012714 (2005). 